() Open Access Full Text Article

ORIGINALRESEARCH

\title{
Gastrin-17 Combined with CEA, CAI2-5 and CA19-9 Improves the Sensitivity for the Diagnosis of Gastric Cancer
}

\section{Zhen Wang $\mathbb{1}^{*}$ \\ Tang-Ming Mo* \\ Lei Tian \\ Jun-Qiang Chen}

Department of Gastrointestinal Surgery, The First Affiliated Hospital of Guangxi Medical University, Nanning, 53002I, Guangxi Zhuang Autonomous Region, People's Republic of China

*These authors contributed equally to this work
Correspondence: Jun-Qiang Chen;

Zhen Wang

Email gxhans@।63.com;

wangzhensurgeon@163.com
Background: Previous studies reported the utility of serum tumor markers (such as CEA, CA12-5 and CA19-9) and gastrin-17 in the diagnosis of gastric cancer (GC). However, the value of these serum markers for diagnosing GC is still under debate. In this study, we aimed to evaluate the effect of gastrin-17, CEA, CA12-5 and CA19-9 in the diagnosis of GC.

Methods: The level of CEA, CA12-5, CA19-9 and gastrin-17 was tested in 230 GC patients and 99 healthy people. The value of the four markers for diagnosing GC was analyzed.

Results: The positive rate of Gastrin-17, CEA, CA19-9 and CA12-5 was much higher in GC group $(22.61 \%, 22.61 \%, 20.00 \%$ and $8.26 \%$, respectively) than that of healthy control group $(5.05 \%, 2.02 \%, 1.01 \%$ and $2.02 \%$, respectively). The sensitivity of Gastrin-17, CEA, CA125 and CA19-9 in the diagnosis of GC was $22.61 \%, 22.61 \%, 6.96 \%$ and $20.00 \%$, respectively, and the corresponding specificity was $94.95 \%, 97.98 \%, 98.99 \%$ and $98.99 \%$, respectively. By using the optimal cut-off value derived from the area under curve (AUC) of receiver operating characteristic curve, the AUC of gastrin-17, CEA, CA12-5, CA19-9 increased to $0.72,0.64,0.61$ and 0.65 , respectively. After combining the four markers, the AUC increased to 0.79 (95\% CI: $0.75-0.84$ ), and the corresponding sensitivity and specificity were $65.22 \%$ (95\% CI: $58.70-71.40 \%$ ) and $84.85 \%$ (95\% CI: 76.20-91.30\%), respectively, which were significantly higher than those of separate markers $(P<0.05)$.

Conclusion: CEA, CA12-5, CA19-9 and gastrin-17 were all valuable in the diagnosis of $\mathrm{GC}$, and gastrin-17 had the best diagnostic value among the four markers. Gastrin-17 combined with CEA, CA12-5 and CA19-9 could improve the diagnostic value of GC significantly. Prospective, multi-center studies are needed to validate our findings.

Keywords: gastric cancer, tumor marker, gastrin, diagnosis

\section{Introduction}

Based on the report of the International Agency for Research on Cancer GLOBOCAN project, gastric cancer (GC) remains the fifth most frequent malignancy and the third leading cause of cancer death worldwide, which is responsible for over 1,000,000 new cases and an estimated 783,000 deaths in 2018, ${ }^{1}$ suggesting more and more people are influenced by this disease. Although great progress has achieved in the treatment of GC, the prognosis of GC patients remains unsatisfactory, especially for those with advanced or metastatic GC. ${ }^{2}$ Therefore, early detection of GC is very vital in clinics. However, detecting GC at early stages is still a huge challenge due to vacancy of specific detection tests. Until now, the confirmation of GC is still based on the pathological examination of specimens 
obtained by gastroscopy. ${ }^{3}$ Nevertheless, the cost of gastroscopic screening is very high and the compliance of patients is poor. Thus, applying gastroscopy and biopsy for large-scale screening of GC is very difficult.

Fortunately, some serum markers were reported to be useful in the detection of GC, including tumor markers and specific molecules secreted by the gastric mucosa, such as Pepsinogen I (PGI), PGII, and gastrin-17 (G-17). ${ }^{4-7}$ Additionally, anti-H. pylori antibodies, which indicate the reaction of gastric mucosa to an exogenous pathogen, have also been studied as a diagnostic marker for GC. ${ }^{6,7}$ Tumor markers such as cancer antigen 12-5 (CA12-5), cancer antigen 19-9 (CA19-9), carbohydrate antigen 72-4 (CA72-4), and carcinoembryonic antigen (CEA) have been used for the detection of kinds of tumors, such as pancreatic cancer and colorectal cancer. ${ }^{8,9}$ However, the results were inconsistent when these markers were used individually for the detection of GC, which meant neither sensitive nor specific tumor markers were identified for GC currently. ${ }^{8,10}$ Recently, some reports found that the combination of tumor markers could distinguish benign tumors from malignant tumors, such as the combination of Galectin-3 and Ki-67 for the diagnosis of thyroid tumors. ${ }^{11}$ And this was confirmed in GC by the combination of CEA, CA12-5 and CA19-9. ${ }^{12}$ Gastrin, a hormone secreted by the $G$ cells of the pyloric glands of the stomach, can induce gastric acid secretion by binding to cholecystokinin-2 (CCK) receptors. Studies found that gastrin could regulate several important cellular processes in the gastric epithelium including invasion, migration, apoptosis, proliferation, angiogenesis and tissue remodeling. ${ }^{5,13}$ Elevated serum gastrin is caused by lots of conditions, including GC, ${ }^{14}$ suggesting gastrin can be used as a potential marker for the diagnosis of GC. Furthermore, some researchers evaluated the value of detecting gastrin-17 levels in serum for pre-cancerous lesion screening in GC, but reports on assessing the effect of gastrin in the diagnosis of GC were very few. ${ }^{15,16}$ Although Tu et al and Wang et al evaluated the diagnostic value of gastrin-17 for GC, they mainly aimed to assess the association between gastrin-17 and GC by using the method of epidemiology survey, not a diagnostic test. ${ }^{17,18}$ Therefore, the effect of gastrin in the diagnosis of GC should be clarified further.

In this study, we evaluated the value of detecting serum gastrin for the diagnosis of GC, and whether the combination of gastrin and tumor markers (CEA, CA12-5 and
CA19-9) would improve the accuracy for the diagnosis of GC.

\section{Methods}

\section{Patients}

The data of 230 patients with GC confirmed by histopathology, who were admitted to the First Affiliated Hospital of Guangxi University from August 2018 to November 2019, were collected and analyzed retrospectively. And the data of 99 healthy people without gastric diseases or other malignant tumors who received physical examination (including gastric endoscopy, chest and abdomen $\mathrm{CT}$, and other routine physical examinations) during the same period at the First Affiliated Hospital of Guangxi Medical University were collected as a control. The inclusion criteria were: 1) all the data of participants were complete, 2) all the GC patients were confirmed by histopathology, and all the healthy people did not have gastric symptoms, history of gastric diseases or malignant tumors. And the exclusion criteria were: 1) Participants who have renal dysfunction, 2) Participants who have proton pump inhibitors or $\mathrm{H} 2$ receptor antagonists within a month before admission, 3) Participants who have a history of other malignant tumors or were pregnant. All procedures performed in this study involving human participants were in accordance with the Declaration of Helsinki (as revised in 2013). And this study was approved by the Ethics Committee of the First Affiliated Hospital of Guangxi Medical University, and written informed consent was obtained from all the participants.

\section{Serum Collection and Testing}

The serum of participants was collected after fasting overnight. For GC patients receiving gastrectomy, the serum was collected preoperatively (less than one month since the diagnosis of GC). The serum level of CEA, CA19-9 and CA12-5 was tested by electrical chemiluminescent immunoassay (ECLIA, ARCHITECT, USA), and the serum level of Gastrin-17 was tested by enzyme-linked immunosorbent (ELISA, commercial kits from Biohit Oyj, Finland). The normal reference range of Gastrin-17, CEA, CA19-9 and CA12-5 in our institution was $1.00-15.00$ $\mathrm{pmol} / \mathrm{L}, 0.00-5.00 \mathrm{ng} / \mathrm{mL}, 0.00-37.00 \mathrm{U} / \mathrm{mL}$ and 0.00 $35.00 \mathrm{U} / \mathrm{mL}$, respectively, which were established according to the instructions. 


\section{Data Collection}

For data analysis, the following data were recorded for all the participants: gender, age, body height, body weight, the serum level of CEA, CA19-9, CA12-5 and Gastrin-17. Additionally, the following information of GC patients were collected: tumor location, tumor size, differentiation degree, and tumor stage (TNM stage). ${ }^{19}$ All the serum testers were blind of the diagnosis of the included patients.

\section{Statistical Analysis}

All the data were expressed as medians and quartiles. The diagnostic value of serum markers was evaluated by using the area under the receiver operating characteristic (ROC) curve (AUC). The AUC was compared by using DeLong test. Multivariate logistic regression analysis was used to establish the diagnostic mathematical model. Comparisons among groups were analyzed by using $t$-tests for continuous variables, and by using Chi-square tests for categorical variables. The sensitivity, specificity, positive predictive value (PPV), and negative predictive value (NPV) (and their 95\% confidence intervals (CIs)) were used for effectiveness evaluation. A value of $P<0.05$ was considered statistically significant. The SPSS 21.0 and MedCalc version 15.2.2 software packages were used to analyze the data.

\section{Results}

The Serum Level of Gastrin- 17, CEA, CAI2-5 and CAI9-9 in Gastric Cancer and Control Groups

Totally, 329 participants (230 GC patients and 99 healthy controls) were included in this study, and the flow diagram was listed as Figure 1. There was no statistical significance

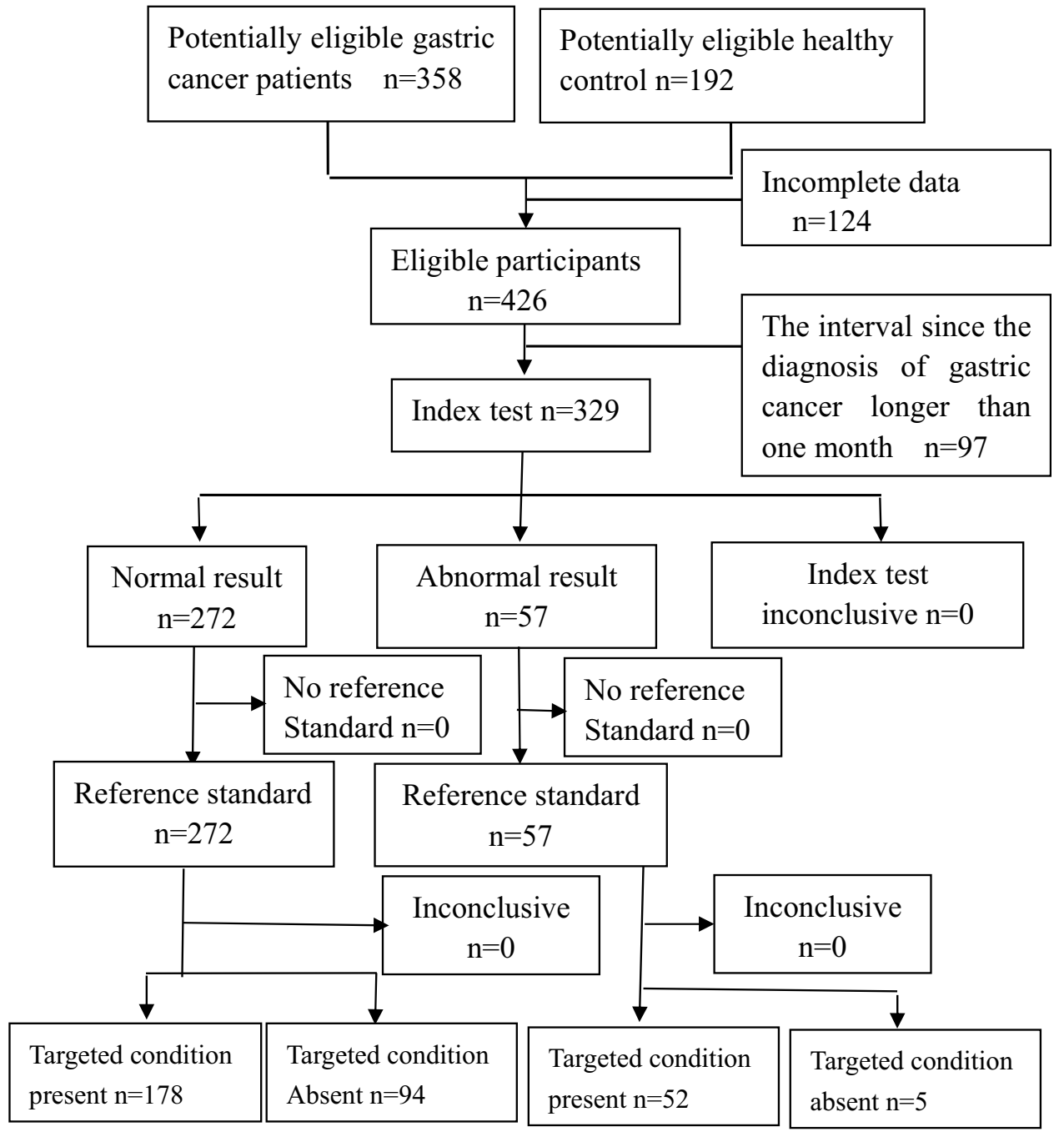

Figure I Flow diagram. 
Table I The Clinical Characteristics of Participants in Gastric Cancer and Control Groups

\begin{tabular}{|l|c|c|c|}
\hline & $\begin{array}{c}\text { GC Group } \\
(\mathbf{n = 2 3 0 )}\end{array}$ & $\begin{array}{c}\text { Control Group } \\
(\mathbf{n}=99)\end{array}$ & P value \\
\hline Age (years) & $57.0(48.0-66.0)$ & $54.0(44.0-63.0)$ & 0.153 \\
Gender (M/F) & $152 / 78$ & $56 / 43$ & 0.100 \\
Height $(\mathrm{cm})$ & $162.0(156.0-168.0)$ & $165.0(158.0-170.0)$. & 0.064 \\
BW $(\mathrm{kg})$ & $55.3(50.0-62.0)$ & $63.5(55.0-72.0)$ & $<0.001$ \\
BMI $\left(\mathrm{kg} / \mathrm{m}^{2}\right)$ & $20.9(19.3-23.6)$ & $23.8(21.4-25.8)$ & $<0.001$ \\
\hline
\end{tabular}

Abbreviations: BW, body weight; BMI, body mass index; GC, gastric cancer; $M$, male; $F$, female.

between the two groups in terms of age, gender and body height $(P>0.05)$. However, the body weight and body mass index (BMI) were much higher in healthy control group than in the GC group $(P<0.05)$, which might be caused by the disease of GC (Table 1).

According to the test results, the median level of Gastrin17, CEA, CA19-9 and CA12-5 were $4.55 \mathrm{pmol} / \mathrm{L}, 2.22 \mathrm{ng} /$ $\mathrm{mL}, 10.00 \mathrm{Uarb} / \mathrm{mL}$ and $11.55 \mathrm{Uarb} / \mathrm{mL}$ in GC group, respectively. And the median level of Gastrin-17, CEA, CA19-9 and CA12-5 were $1.95 \mathrm{pmol} / \mathrm{L}, 1.76 \mathrm{ng} / \mathrm{mL}, 4.51$ $\mathrm{Uarb} / \mathrm{mL}$ and $9.40 \mathrm{Uarb} / \mathrm{mL}$ in healthy control group, respectively, which were all much lower than that in GC group $(P<$ 0.05 , Table 2). According to the normal reference range of
Gastrin-17, CEA, CA19-9 and CA12-5 in our institution, the positive rate of Gastrin-17, CEA, CA19-9 and CA12-5 were $22.61 \%, 22.61 \%, 20.00 \%$ and $8.26 \%$ in GC group, respectively; and were $5.05 \%, 2.02 \%, 1.01 \%$ and $2.02 \%$ in healthy control group, respectively, suggesting much lower positive rates in healthy control group $(P<0.05$, Table 2$)$.

\section{The Value of Serum Gastrin- I7, CEA, CAI2-5 and CA19-9 in the Diagnosis of Gastric Cancer}

Firstly, we used the normal reference range of our institution as the cutoff values, as a result, we found that the sensitivity of Gastrin-17, CEA, CA12-5 and CA19-9 in the diagnosis of GC were $22.61 \%$ (95\% CI: $17.40-8.60 \%), 22.61 \%$ (95\% CI: 17.40-28.60\%), 6.96\% (95\% CI: $4.00-11.10 \%$ ) and $20.00 \%$ (95\% CI: 15.00-25.80\%), respectively, and the corresponding specificity were $94.95 \%$ (95\% CI: $88.60-98.30 \%$ ), 97.98\% (95\% CI: 92.90-99.80\%), 98.99\% (95\% CI: 94.50-100.00\%) and $98.99 \%$ (95\% CI: $94.50-100.00 \%$ ), respectively (Table 3). This suggested that Gastrin-17 had a comparable value as CEA in the diagnosis of GC, which was better than CA12-5 and CA19-9.

Then, we derived cutoff values from the ROC by using the MedCalc statistical software as the diagnostic cutoff

Table 2 The Level and Positive Rate of Serum Markers in Gastric Cancer and Control Groups

\begin{tabular}{|c|c|c|c|c|c|}
\hline & \multicolumn{2}{|l|}{ GC Group } & \multicolumn{2}{|l|}{ Control Group } & \multirow[t]{2}{*}{$P$ value } \\
\hline & Serum Level & Positive N (\%) & Serum Level & Positive N (\%) & \\
\hline G-I7 (pmol/L) & $4.55(2.08-13.96)$ & $52(22.6 I)$ & $1.95(1.08-3.85)$ & $5(5.05)$ & $<0.001$ \\
\hline CEA (ng/mL) & $2.22(1.36-4.77)$ & $52(22.6 I)$ & $1.76(1.13-2.36)$ & $2(2.02)$ & $<0.001$ \\
\hline CAI9-9 (Uarb/mL) & $10.00(3.30-24.21)$ & $46(20.00)$ & 4.5। (2.33-9.39) & I (I.0I) & $<0.001$ \\
\hline CAI2-5 (Uarb/mL) & II.55 (8.20-17.28) & $19(8.26)$ & $9.40(6.90-13.50)$ & $2(2.02)$ & 0.002 \\
\hline
\end{tabular}

Abbreviations: CEA, carcinoembryonic antigen; CAI2-5, cancer antigen 12-5; CAI9-9, cancer antigen 19-9; G-17, gastrin-17; GC, gastric cancer.

Table 3 The Diagnostic Value of Gastrin-17, CEA, CA12-5 and CA19-9 in Gastric Cancer

\begin{tabular}{|l|c|c|c|c|c|}
\hline & Cut-off Value & Sen (\%) & 95\% Cl (\%) & Spe (\%) & 95\% CI (\%) \\
\hline G-I7 & $15.00 \mathrm{pmol} / \mathrm{L}$ & 22.61 & $17.40-28.60$ & 94.95 & $88.60-98.30$ \\
& $2.73 \mathrm{pmol} / \mathrm{L}$ & 71.30 & $65.00-77.10$ & 63.64 & $53.40-73.10$ \\
\hline CEA & $5.00 \mathrm{ng} / \mathrm{mL}$ & 22.61 & $17.40-28.60$ & 97.98 & $92.90-99.80$ \\
& $3.38 \mathrm{ng} / \mathrm{mL}$ & 34.78 & $28.60-41.30$ & 93.94 & $87.30-97.70$ \\
\hline CAI25 & $35.00 \mathrm{Uarb} / \mathrm{mL}$ & 6.96 & $4.00-11.10$ & 98.99 & $94.50-100.00$ \\
& $9.70 \mathrm{Uarb} / \mathrm{mL}$ & 66.09 & $59.60-72.20$ & 53.54 & $43.20-63.60$ \\
\hline \multirow{2}{*}{ CAI99 } & $37.00 \mathrm{Uarb} / \mathrm{mL}$ & 20.00 & $15.00-25.80$ & 98.99 & $94.50-100.00$ \\
& $9.39 \mathrm{Uarb} / \mathrm{mL}$ & 52.61 & $45.90-59.20$ & 75.76 & $66.10-83.80$ \\
\hline
\end{tabular}

Abbreviations: CEA, carcinoembryonic antigen; CAI2-5, cancer antigen 12-5; CAI9-9, cancer antigen 19-9; Cl, confidence interval; G-17, gastrin-17; Sen, sensitivity; Spe, specificity. 
values for Gastrin-17, CEA, CA12-5 and CA19-9, which were $2.73 \mathrm{pmol} / \mathrm{L}, 3.38 \mathrm{ng} / \mathrm{mL}, 9.70 \mathrm{Uarb} / \mathrm{mL}$, and 9.39 $\mathrm{Uarb} / \mathrm{mL}$, respectively. By using the optimal cutoff values, we found that the sensitivity of Gastrin-17, CEA, CA12-5 and CA19-9 in the diagnosis of GC were $71.30 \%$ (95\% CI: 65.00-77.10\%), 34.78\% (95\% CI: 28.60-41.30\%), 66.09\% (95\% CI: 59.60-72.20\%) and 52.61\% (95\% CI: 45.90 $59.20 \%$ ), respectively, and the corresponding specificity were 63.64\% (95\% CI: 53.40-73.10\%), 93.94\% (95\% CI: 87.30-97.70\%), 53.54\% (95\% CI: 43.20-63.60\%) and 75.76\% (95\% CI: 66.10-83.80\%), respectively (Table 3). The PPV of Gastrin-17, CEA, CA12-5 and CA19-9 were 82.00 (95\% CI: 77.60-85.70), 93.00 (95\% CI: 85.80-96.70), 76.80 (95\% CI: 72.40-80.60), and 83.40 (95\% CI: 77.70 $87.90)$, respectively, and the corresponding NPV were 48.80 (95\% CI: 42.60-55.10), 38.30 (95\% CI: 35.80-40.80), 40.50 (95\% CI: 34.40-46.80), and 40.80 (95\% CI: 36.60-45.10), respectively (Table 4$)$.

\section{Gastrin-17 Combined with CEA, CAI2-5 and CA 19-9 Improves the Diagnostic Value of Gastric Cancer}

In order to compare the diagnostic efficiency of separate and combined tests, we calculated the AUC of ROC curve. As a result, the AUC of G17, CEA, CA12-5, CA19-9 were 0.72 (95\% CI: 0.67-0.77), 0.64 (95\% CI: 0.58-0.69), 0.61 (95\% CI: 0.55-0.66) and 0.65 (95\% CI: 0.59-0.70), respectively, which showed that $\mathrm{G} 17$ had the best diagnostic efficiency among the four markers, although it was not statistically significant compared with CEA and CA19-9 (Table 5). By combining the four markers, the AUC increased to 0.79 (95\% CI: $0.75-0.84$ ), and the corresponding sensitivity and specificity were $65.22 \%$ (95\% CI: 58.70-71.40\%) and 84.85\% (95\% CI: 76.20-91.30\%), respectively. This suggested that Gastrin-17 combined with CEA, CA12-5 and CA19-9 could improve the diagnostic accuracy of GC significantly $(P<0.05)$ (Figure 2, Table 5).
Table 5 The Comparison of AUC Among the Serum Tumor Markers

\begin{tabular}{|l|c|c|}
\hline Comparisons & z Statistic & P value \\
\hline GI7 vs CEA & 1.921 & 0.0548 \\
GI7 vs CAI2-5 & 2.455 & 0.0141 \\
GI7 vs CAI9-9 & 1.643 & 0.1004 \\
GI7 vs Combined test & 2.911 & 0.0036 \\
\hline
\end{tabular}

Abbreviations: AUC, the area under the receiver operating characteristic curve; CEA, carcinoembryonic antigen; CAl2-5, cancer antigen 12-5; CAl9-9, cancer antigen 19-9; GI7, gastrin-17.

\section{The Association of Serum Gastrin-I7 and Clinicopathologic Characteristics of Gastric Cancer}

In the $230 \mathrm{GC}$ patients, 152 patients $(66.1 \%)$ were male and 78 patients $(33.9 \%)$ were female. One hundred and ninety-eight patients $(74.3 \%)$ were older than 40 years old, and only 32 patients (25.7\%) were younger than 40 years old. Most of the patients had advanced stages with lymph node metastasis. The clinicopathologic characteristics of the included patients are summarized in Table 6 .

In order to explore the relationship of serum gastrin-17 and clinicopathologic characteristics of gastric cancer, we used the method of univariate analysis. As a result, we found that GC patients with distant metastasis (M1) had a higher level of serum gastrin-17 $(P<0.05$, Table 6).

\section{Discussion}

Gastric cancer remains one of the most common malignant tumors with high mortality worldwide. Early diagnosis and timely treatment are the most efficient methods to improve the prognosis. Serum tumor markers are often used for early diagnosis, treatment effect assessment and disease monitoring of tumors. ${ }^{20}$ Unfortunately, the optimal serum tumor marker for the timely detection of GC remains under investigation. ${ }^{21}$ Recently, several studies reported the utility of serum tumor markers (such as CEA, CA12-

Table 4 Comparison of Diagnostic Efficiency Between Separate and Combined Tests in Gastric Cancer

\begin{tabular}{|l|c|c|c|c|c|}
\hline & AUC (95\% Cl) & Sen $\mathbf{( 9 5 \% ~ C l )}$ & Spe (95\% CI) & PPV (95\% CI) & NPV (95\% CI) \\
\hline GI7 & $0.72(0.67-0.77)$ & $71.30(65.00-77.10)$ & $63.64(53.40-73.10)$ & $82.00(77.60-85.70)$ & $48.80(42.60-55.10)$ \\
CEA & $0.64(0.58-0.69)$ & $34.78(28.60-41.30)$ & $93.94(87.30-97.70)$ & $93.00(85.80-96.70)$ & $38.30(35.80-40.80)$ \\
CAI25 & $0.61(0.55-0.66)$ & $66.09(59.60-72.20)$ & $53.54(43.20-63.60)$ & $76.80(72.40-80.60)$ & $40.50(34.40-46.80)$ \\
CA199 & $0.65(0.59-0.70)$ & $52.61(45.90-59.20)$ & $75.76(66.10-83.80)$ & $83.40(77.70-87.90)$ & $40.80(36.60-45.10)$ \\
Combined test & $0.79(0.75-0.84)$ & $65.22(58.70-71.40)$ & $84.85(76.20-91.30)$ & $90.90(86.10-94.10)$ & $51.20(46.30-56.10)$ \\
\hline
\end{tabular}

Abbreviations: AUC, the area under the receiver operating characteristic curve; CEA, carcinoembryonic antigen; CAI2-5, cancer antigen 12-5; CAI9-9, cancer antigen 19-9; Cl, confidence interval; G-17, gastrin-17; NPV, negative predictive value; PPV, positive predictive value; Sen, sensitivity; Spe, specificity. 


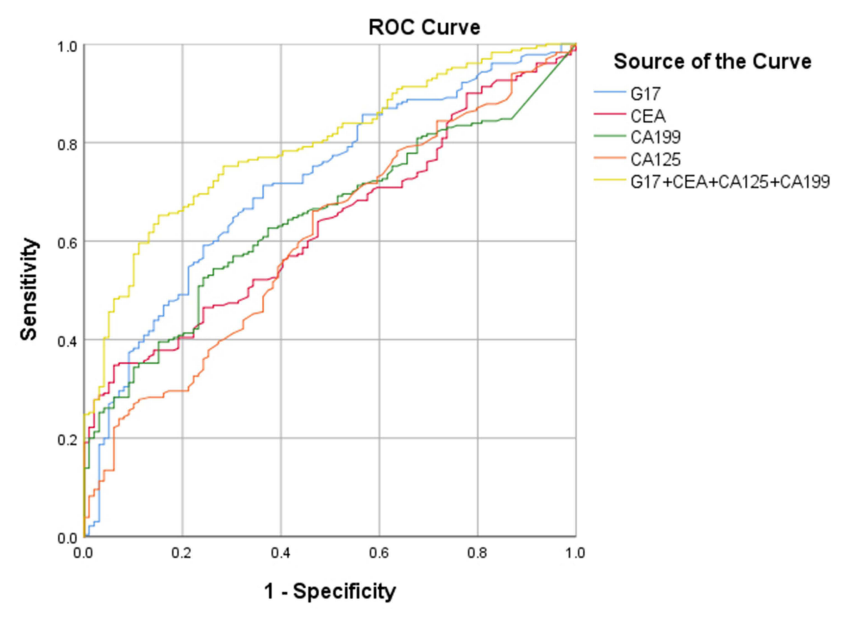

Figure 2 The receiver operating characteristic (ROC) curve of gastrin-17, CEA, CAI2-5, CAI9-9 and the combined tests in the diagnosis of gastric cancer.

5 and CA19-9) in the diagnosis of GC, and some researchers found that gastrin-17 was closely associated with GC. However, the value of these serum markers for diagnosing GC is still under debate. Therefore, we evaluated the value of the four serum markers (CEA, CA12-5, CA19-9 and gastrin-17) for diagnosing GC in this study. After analyzing the data of $230 \mathrm{GC}$ patients and 99 healthy people, we found that gastrin-17 was most valuable among the four markers in the diagnosis of GC, and gastrin-17 combined with CEA, CA12-5 and CA19-9 could improve the diagnostic accuracy of GC significantly.

CEA is an oncofetal protein related with cell adhesion and the inhibition of apoptosis, whose main clinical use is in colorectal cancer patients. ${ }^{22}$ CA19-9 is a specific marker of digestive system tumors and widely used in the monitoring and diagnosis of pancreatic cancer. ${ }^{23}$ CA12-5 was first detected in ovarian cancer and had been reported to be positive in many malignant tumors, including GC. ${ }^{24}$ Recently, CEA, CA12-5 and CA19-9 have become the most commonly used tumor markers for diagnosing $\mathrm{GC}$ preoperatively. But previous studies found that the positive rate of CEA, CA12-5 and CA19-9 in GC were 4.4-21.1\%, $6.7-42.4 \%$ and $11.7-27.8 \%$, respectively, suggesting little benefit to screen primary GC, especially for early GC. ${ }^{4,25,26}$ However, few studies focused on the diagnostic value of the three markers in GC by using normatively statistical methods, not reporting the data of sensitivity or specificity. In this study, we found that the positive rate of CEA, CA12-5 and CA19-9 was 22.61\%, 8.26\% and $20.00 \%$, respectively, which was similar with previous studies. $^{25,26}$ Further analysis indicated that the sensitivity of the three makers was very low (Table 3), and this suggested that the value of the three markers was very limited in the diagnosis of GC. However, the diagnostic accuracy was improved when we used the cutoff values derived from the ROC, and all the cutoff values were lower than we used in clinics (Table 3). This demonstrated that lowering the cutoff values of the three makers appropriately might be helpful for improving the value in the diagnosis of GC, but the optimal cutoff values should be established by studies with larger sample size in future.

Gastrin, which is an agonist to induce the secretion of gastric acid, takes part in many important cellular processes in the gastric epithelium. ${ }^{5}$ The level of gastrin is affected by lots of factors, such as atrophic gastritis, H. pylori infection, and acid suppression. Therefore, it is considered as an accurate functional marker of the state of gastric mucosa. ${ }^{27}$ Recently, some researchers found that hypergastrinemia, particularly that accompanied with chronic $\mathrm{H}$ pylori infection, may be associated with the development of GC. ${ }^{5}$ Theoretically, testing of serum gastrin concentration is reasonably simple, and several studies have assessed whether the level of serum gastrin assists in the diagnosis, helps to determine the respectability, or predicts the prognosis of GC. ${ }^{28}$ Although a much higher level of serum gastrin has been demonstrated in patients with GC, the attempts to use serum gastrin concentration as a marker for the diagnosis of GC failed. ${ }^{29}$ And the clinical usefulness of such assessment was limited in past several years. However, recent studies found that gastrin was closely associated with GC and precancerous lesions. ${ }^{16,30-32}$ Moreover, Yu et al found that serum gastrin level could be used for screening pre-cancerous lesion in gastric cancer. ${ }^{33}$ These suggested the possible role of gastrin in the diagnosis of GC. In order to assess the value of gastrin in the diagnosis of $\mathrm{GC}, \mathrm{Tu}$ et al and Wang et al conducted cohort studies using the method of epidemiology survey. ${ }^{17,18}$ Although they got the data of diagnostic accuracy, the reliability is relatively low because of the method and very few GC patients. ${ }^{17,18}$ In our studies, we found that the positive rate of gastrin-17 was $22.61 \%$ in GC, but the sensitivity of gastrin-17 could reach to $71.30 \%$ (95\% CI: $65.00-77.10 \%$ ) for diagnosing GC after using an optimal cutoff value. This demonstrated that gastrin-17 could be used as a surrogate marker for the diagnosis of GC. Our results were similar with the study by Murphy et $\mathrm{al}^{16}{ }^{16}$ who found that gastrin-17 concentrations were significantly associated with the increased risk of GC. Additionally, our study showed that GC patients with distant metastasis (M1) had a much higher level of 
Table 6 The Associations Between the Level of Gastrin- 17 and the Characteristics of Gastric Cancer

\begin{tabular}{|c|c|c|c|}
\hline & $\mathbf{N}(\%)$ & G-I7 (pmol/L) & $P$ value \\
\hline Age & & & 0.489 \\
\hline$\leq 40$ & $32(13.9)$ & $4.45(2.15-7.95)$ & \\
\hline $41-65$ & $139(60.4)$ & $4.56(2.1|-| 4.82)$ & \\
\hline$\geq 66$ & $59(25.7)$ & $4.43(1.7 \mid-14.38)$ & \\
\hline Gender & & & 0.089 \\
\hline Male & $152(66.1)$ & $4.18(1.94-12.33)$ & \\
\hline Female & 78 (33.9) & $5.32(2.76-18.72)$ & \\
\hline Tumor diameter & & & 0.260 \\
\hline$<5 \mathrm{~cm}$ & $159(69.1)$ & $4.54(1.94-13.21)$ & \\
\hline$\geq 5 \mathrm{~cm}$ & 71 (30.9) & $4.56(2.29-14.52)$ & \\
\hline Tumor location & & & 0.195 \\
\hline Cardia & $9(3.9)$ & 8.38 (4.39-24.37) & \\
\hline Fundus & $15(6.5)$ & $5.92(1.95-15.63)$ & \\
\hline Body & $51(22.2)$ & $7.62(2.37-21.83)$ & \\
\hline Antrum & 147 (63.9) & $4.03(1.94-10.10)$ & \\
\hline Diffused & $8(3.5)$ & $6.78(2.4 I-20.99)$ & \\
\hline Differentiation & & & 0.164 \\
\hline Poorly & $157(68.3)$ & $4.50(1.89-12.90)$ & \\
\hline Moderately & $70(30.4)$ & $5.78(2.31-18.39)$ & \\
\hline Well & $3(1.3)$ & 4.43 (4.28-) & \\
\hline Infiltration depth & & & 0.394 \\
\hline $\mathrm{TI}$ & $25(10.9)$ & $3.67(1.88-10.80)$ & \\
\hline $\mathrm{T} 2$ & $40(17.4)$ & 4.91 (1.77-22.78) & \\
\hline T3 & $107(46.5)$ & $4.56(2.17-15.57)$ & \\
\hline $\mathrm{T} 4$ & $58(25.2)$ & $4.44(2.17-12.77)$ & \\
\hline Lymph node metastasis & & & 0.158 \\
\hline No & $60(26.1)$ & $4.6 \mathrm{I}(\mathrm{I} .84-10.36)$ & \\
\hline $\mathrm{NI}-3$ & $170(73.9)$ & $4.55(2.27-15.59)$ & \\
\hline Distant metastasis & & & 0.049 \\
\hline Mo & $216(93.9)$ & $4.38(1.96-12.52)$ & \\
\hline MI & $14(6.1)$ & II.23 (7.03-24.92) & \\
\hline Pathological stage & & & 0.175 \\
\hline I & $40(17.4)$ & $3.32(1.66-11.15)$ & \\
\hline ॥ & $63(27.4)$ & $5.39(3.14-14.38)$ & \\
\hline III & $113(49.1)$ & 4.34 (1.98-13.23) & \\
\hline IV & $14(6.1)$ & II.23 (7.03-24.92) & \\
\hline
\end{tabular}

Abbreviation: G-17, gastrin-17.

serum gastrin-17, suggesting that the level of gastrin-17 could be affected by the clinicopathological characteristics of GC. And this was consistent with the study by Zhao et $\mathrm{al},{ }^{32}$ implying that the clinicopathological characteristics of GC should be considered when using gastrin-17 as a diagnostic marker.

Although tumor markers such as CEA, CA12-5 and CA19-9 have been widely used in the diagnosis of kinds of tumors, including GC, the value remains being questioned because of wide variance in diagnostic accuracy. ${ }^{26,34}$ In our study, we found that the sensitivity of the four markers for diagnosing $\mathrm{GC}$ was less than $25 \%$ (Table 3), which could not meet the requirement of clinical practice. By using the optimum cut-off values obtained from the logistic regression analysis and the ROC curve, the sensitivity of the four markers increased significantly (Table 3 ). And 
the combination of the four tumor markers could improve the diagnostic accuracy of GC further, with a sensitivity of $65.22 \%$ and an AUC of 0.79 (Table 4). This suggested that combined detection of tumor markers could improve the diagnostic value of GC, which was consistent with previous studies. ${ }^{12,35}$

In fact, there are some limitations for our study. Firstly, the data in our study were from a single center in China, and were analyzed retrospectively. This decreased the representativeness and reliability of the results. Secondly, there was no control group including patients with benign gastric lesions, and the results were not yet validated in a prospective group. At last, we did not compare the diagnostic value of the four serum markers in GC. Therefore, we should view the results of this study prudently, and prospective, multi-center studies are needed to validate the results.

In conclusion, CEA, CA12-5, CA19-9 and gastrin-17 were all useful in the preoperative diagnosis of GC, and gastrin-17 was the most accurate one among the four markers. Gastrin-17 combined with CEA, CA12-5 and CA19-9 could improve the diagnostic value of GC significantly.

\section{Data Sharing Statement}

All the data used to support the findings of this study are available from the corresponding author upon request.

\section{Statement of Ethics}

All procedures performed in this study involving human participants were in accordance with the Declaration of Helsinki (as revised in 2013). This study was approved by the Ethics Committee of the First Affiliated Hospital of Guangxi Medical University, and written informed consent was obtained from all the participants.

\section{Acknowledgments}

The authors thank colleagues from the First Affiliated Hospital of Guangxi Medical University for their warmhearted assistance in developing this paper. The manuscript is as a preprint version in Research Square (https:// www.researchsquare.com/article/rs-390531/v1).

\section{Author Contributions}

All authors contributed to data analysis, drafting or revising the article, gave final approval of the version to be published, agreed to the submitted journal, and agree to be accountable for all aspects of the work.

\section{Funding}

This manuscript was supported by the National Natural Science Foundation of China (82002492) and the Natural Science Foundation of Guangxi (2018GXNSFBA281159).

\section{Disclosure}

The authors have no conflicts of interest to declare.

\section{References}

1. Bray F, Ferlay J, Soerjomataram I, et al. Global cancer statistics 2018: GLOBOCAN estimates of incidence and mortality worldwide for 36 cancers in 185 countries. CA Cancer J Clin. 2018;68(6): 394-424. doi:10.3322/caac. 21492

2. Thrift AP, El-Serag HB. Burden of gastric cancer. Clin Gastroenterol Hepatol. 2020;18(3):534-542. doi:10.1016/j.cgh.2019.07.045

3. Agnes A, Biondi A, Laurino A, et al. Global updates in the treatment of gastric cancer: a systematic review. Part 1: staging, classification and surgical treatment. Updates Surg. 2020;72(2):341-353. doi:10. 1007/s13304-020-00736-3

4. Feng F, Tian Y, Xu G, et al. Diagnostic and prognostic value of CEA, CA19-9, AFP and CA125 for early gastric cancer. BMC Cancer. 2017;17(1):737. doi:10.1186/s12885-017-3738-y

5. Burkitt MD, Varro A, Pritchard DM. Importance of gastrin in the pathogenesis and treatment of gastric tumors. World J Gastroenterol. 2009;15(1):1-16. doi:10.3748/wjg.15.1

6. Watabe H, Mitsushima T, Yamaji Y, et al. Predicting the development of gastric cancer from combining Helicobacter pylori antibodies and serum pepsinogen status: a prospective endoscopic cohort study. Gut. 2005;54(6):764-768. doi:10.1136/gut.2004.055400

7. Yamaguchi Y, Nagata Y, Hiratsuka R, et al. Gastric cancer screening by combined assay for serum Anti-Helicobacter pylori IgG antibody and serum pepsinogen levels - the ABC method. Digestion. 2016;93 (1):13-18. doi:10.1159/000441742

8. He CZ, Zhang KH, Li Q, et al. Combined use of AFP, CEA, CA125 and CA19-9 improves the sensitivity for the diagnosis of gastric cancer. BMC Gastroenterol. 2013;13(1):87. doi:10.1186/1471-230X13-87

9. Lin Z, Bian H, Chen C, Chen W, Li Q. Application of serum pepsinogen and carbohydrate antigen 72-4 (CA72-4) combined with gastrin-17 (G-17) detection in the screening, diagnosis, and evaluation of early gastric cancer. $J$ Gastrointest Oncol. 2021;12 (3):1042-1048. doi:10.21037/jgo-21-254

10. Li Y, Yang Y, Lu M, et al. Predictive value of serum CEA, CA19-9 and CA72-4 in early diagnosis of recurrence after radical resection of gastric cancer. Hepatogastroenterology. 2011;58(112):2166-2170.

11. Cui W, Lu X, Zheng S, et al. The use of a combination of Ki-67, Galectin-3, and PTTG can distinguish the benign and malignant thyroid tumor. Clin Lab. 2012;58(5-6):419-426.

12. Yang AP, Liu J, Lei HY, et al. CA72-4 combined with CEA, CA125 and CA19-9 improves the sensitivity for the early diagnosis of gastric cancer. Clin Chim Acta. 2014;437:183-186. doi:10.1016/j.cca.2014. 07.034

13. Maddalo G, Spolverato Y, Rugge M, et al. Gastrin: from pathophysiology to cancer prevention and treatment. Eur $J$ Cancer Prev. 2014;23(4):258-263. doi:10.1097/CEJ.0000000000000008

14. Hayakawa Y, Chang W, Jin G, et al. Gastrin and upper GI cancers. Curr Opin Pharmacol. 2016;31:31-37. doi:10.1016/j.coph.2016. 08.013

15. Wang Y, Zhu Z, Liu Z, et al. The value of detecting pepsinogen and gastrin-17 levels in serum for pre-cancerous lesion screening in gastric cancer. $J$ Int Med Res. 2020;48(3):300060520914826. 
16. Murphy G, Abnet CC, Choo-Wosoba H, et al. Serum gastrin and cholecystokinin are associated with subsequent development of gastric cancer in a prospective cohort of Finnish smokers. Int J Epidemiol. 2017;46(3):914-923. doi:10.1093/ije/dyx030

17. Tu H, Sun L, Dong $X$, et al. A serological biopsy using five stomach-specific circulating biomarkers for gastric cancer risk assessment: a multi-phase study. Am J Gastroenterol. 2017;112(5): 704-715. doi:10.1038/ajg.2017.55

18. Wang Y, Zhu Z, Liu Z, et al. Diagnostic value of serum pepsinogen I, pepsinogen II, and gastrin-17 levels for population-based screening for early-stage gastric cancer. J Int Med Res. 2020;48(3):30 0060520914826.

19. Amin MB, Greene FL, Edge SB, et al. The eighth edition AJCC cancer staging manual: continuing to build a bridge from a population-based to a more "personalized" approach to cancer staging. CA Cancer J Clin. 2017;67(2):93-99. doi:10.3322/caac.21388

20. Rodríguez-Enríquez S, Pacheco-Velázquez SC, Gallardo-Pérez JC, et al. Multi-biomarker pattern for tumor identification and prognosis. $J$ Cell Biochem. 2011;112(10):2703-2715. doi:10.1002/jcb.23224

21. Liang Y, Wang W, Fang C, et al. Clinical significance and diagnostic value of serum CEA, CA19-9 and CA72-4 in patients with gastric cancer. Oncotarget. 2016;7(31):49565-49573. doi:10.18632/oncotarget.10391

22. Basbug M, Arikanoglu Z, Bulbuller N, et al. Prognostic value of preoperative CEA and CA 19-9 levels in patients with colorectal cancer. Hepatogastroenterology. 2011;58(106):400-405.

23. Lamerz R. Role of tumour markers, cytogenetics. Ann Oncol. 1999;10(Suppl 4):145-149. doi:10.1093/annonc/10.suppl_4.S145

24. Klug TL, Bast RC Jr, Niloff JM, et al. Monoclonal antibody immunoradiometric assay for an antigenic determinant (CA 125) associated with human epithelial ovarian carcinomas. Cancer Res. 1984;44 (3):1048-1053

25. Wang W, Chen XL, Zhao SY, et al. Prognostic significance of preoperative serum CA125, CA19-9 and CEA in gastric carcinoma. Oncotarget. 2016;7(23):35423-35436. doi:10.18632/oncotarget.8770

26. Shimada H, Noie T, Ohashi M, et al. Clinical significance of serum tumor markers for gastric cancer: a systematic review of literature by the task force of the Japanese Gastric Cancer Association. Gastric Cancer. 2014;17(1):26-33. doi:10.1007/s10120-013-0259-5
27. Väänänen $H$, Vauhkonen $M$, Helske $T$, et al. Non-endoscopic diagnosis of atrophic gastritis with a blood test. Correlation between gastric istology and serum levels of gastrin-17 and pepsinogen I: a multicentre study. Eur J Gastroenterol Hepatol. 2003;15 (8):885-891. doi:10.1097/00042737-200308000-00009

28. Soran A, Aslar AK, Cöl C. Are preoperative serum gastrin levels related to resectability and survival in gastric cancer? Int $\mathrm{J}$ Clin Pract. 2000;54(10):652-653.

29. Rakic S, Milicevic MN. Serum gastrin levels in patients with intestinal and diffuse type of gastric cancer. Br J Cancer. 1991;64(6):1189. doi:10.1038/bjc.1991.489

30. Kang JM, Kim N, Yoo JY, et al. The role of serum pepsinogen and gastrin test for the detection of gastric cancer in Korea. Helicobacter. 2008;13(2):146-156. doi:10.1111/j.1523-5378.2008.00592.x

31. Mansour-Ghanaei F, Joukar F, Rajpout Y, et al. Screening of precancerous gastric lesions by serum pepsinogen, Gastrin-17, anti-helicobacter pylori and anti- caga Antibodies in dyspeptic patients over 50 years old in Guilan Province, North of Iran. Asian Pac J Cancer Prev. 2014;15(18):7635-7638. doi:10.7314/APJCP.20 14.15.18.7635

32. Zhao WX, Liu ZF, Li XL, et al. Correlations of serum homocysteine, VEGF and gastrin 17 with gastric cancer and precancerous lesions. Eur Rev Med Pharmacol Sci. 2019;23(10):4192-4198.

33. Yu G, Wang GX, Wang HG, et al. The value of detecting pepsinogen and gastrin-17 levels in serum for pre-cancerous lesion screening in gastric cancer. Neoplasma. 2019;66(4):637-640. doi:10.4149/neo_20 $18 \_180825 \mathrm{~N} 647$

34. Gadde R, Tamariz L, Hanna M, et al. Metastatic gastric cancer (MGC) patients: can we improve survival by metastasectomy? A systematic review and meta-analysis. J Surg Oncol. 2015;112 (1):38-45. doi:10.1002/jso.23945

35. Chen C, Chen Q, Zhao Q, et al. Value of combined detection of serum CEA, CA72-4, CA19-9, CA15-3 and CA12-5 in the diagnosis of gastric cancer. Ann Clin Lab Sci. 2017;47(3):260-263.
International Journal of General Medicine

\section{Publish your work in this journal}

The International Journal of General Medicine is an international, peer-reviewed open-access journal that focuses on general and internal medicine, pathogenesis, epidemiology, diagnosis, monitoring and treatment protocols. The journal is characterized by the rapid reporting of reviews, original research and clinical studies across all disease areas. The manuscript management system is completely online and includes a very quick and fair peer-review system, which is all easy to use. Visit http://www.dovepress.com/ testimonials.php to read real quotes from published authors. 